\title{
CONGELABILIDADE DO SÊMEN SUÍNO DE ACORDO COM O PERÍODO DE EQUILÍBRIO PRÉ-CONGELAMENTO E DA SENSIBILIDADE AO RESFRIAMENTO
}

\section{(Freezability of swine semen according to equilibration time and chilling sensitivity)}

\author{
OHATA, P.M.'; BERNARDI, M.L. ${ }^{2}$; REIS, G.R. ${ }^{1}$; BORTOLOZZO, F.P.'; WENTZ, I. ${ }^{1}$
}

${ }^{1}$ Faculdade de Veterinária/UFRGS; ${ }^{2}$ Departamento de Zootecnia, Faculdade de Agronomia/UFRGS;

\begin{abstract}
RESUMO - O objetivo deste estudo foi avaliar a influência do período de equilíbrio pré-congelamento e da sensibilidade ao resfriamento sobre a qualidade do sêmen suíno congelado. Foram utilizados dez ejaculados de cada um dos 8 machos doadores. A motilidade de doses de sêmen preparadas a partir dos mesmos ejaculados utilizados no congelamento, foi avaliada simultaneamente, durante o resfriamento a $15^{\circ} \mathrm{C}$, a cada $24 \mathrm{~h}$. A porção de cada ejaculado destinada ao congelamento foi separada em duas partes que foram submetidas a equilíbrio prévio, a $20^{\circ} \mathrm{C}$, curto $(1,5 \mathrm{~h})$ ou longo $(20 \mathrm{~h})$. As palhetas foram descongeladas a $37^{\circ} \mathrm{C}$ por 20 segundos e seu conteúdo $(0,5 \mathrm{~mL})$ foi diluído (1:6) em Beltsville Thawing Solution (BTS). Foram avaliados a motilidade (MOT), acrossomos normais após o descongelamento (NARPD) e após o teste de termoresistência (NARTTR), e membranas íntegras (MI). Para a análise estatística, os ejaculados foram separados, de acordo com sua sensibilidade ao resfriamento, em dois grupos: motilidade $<60 \%$ (grupo E1) e motilidade $\geq 60 \%$ (grupo E2), no quinto dia de armazenamento a $15^{\circ} \mathrm{C}$. Ejaculados com diferente sensibilidade ao resfriamento apresentaram resposta similar ao congelamento $(P>0,05)$. O equilíbrio por $20 \mathrm{~h}$ não apresentou diferenças na $\mathrm{MOT}$ e $\mathrm{MI}(\mathrm{P}>0,05)$, mas o NARPD e NARTTR foram superiores $(P<0,05)$, em comparação a $1,5 \mathrm{~h}$. Não há associação entre a sensibilidade ao resfriamento, com base na avaliação da motilidade, e a viabilidade do sêmen após o congelamento. Um maior período de equilíbrio (20h), antes do congelamento, permite manter maior integridade do acrossomo, após descongelamento.
\end{abstract}

Palavras-chave: sêmen, suíno, congelamento, espermatozóide, acrossomo

ABSTRACT - The aim of this study was to evaluate the influence of the equilibration time and chilling sensitivity on the quality of frozen swine semen. Ten ejaculates from each of 8 boars were used. Motility of semen doses prepared from the same ejaculates submitted to freezing and stored at $15^{\circ} \mathrm{C}$ was simultaneously evaluated, at $24 \mathrm{~h}$ intervals. From each ejaculate the portion destined to freezing was separated in two parts which were submitted to a short $(1.5 \mathrm{~h})$ or long $(20 \mathrm{~h})$ equilibration period, at $20^{\circ} \mathrm{C}$. The straws were thawed at $37^{\circ} \mathrm{C}$ for 20 seconds and the content $(0,5 \mathrm{~mL})$ was extended in Beltsville Thawing Solution (BTS) (1:6). Motility (MOT), normal acrosomes, after both thawing (NARPD) and thermoresistance test (NARTTR), and membrane integrity (MI) were evaluated. For statistical analysis, the ejaculates were separated according to their cooling sensitivity, in two groups: motility $<60 \%$ (group $\mathrm{E} 1$ ) and $\geq 60 \%$ (group E2), at the fifth day of storage at $15^{\circ} \mathrm{C}$. Ejaculates with different chilling sensitivity showed a similar response to freezing $(P>0.05)$. There were no differences in MOT and Ml, but 20h equilibration was associated with an increase $(P<0.05)$ in NARPD and NARTTR, in comparison to 1.5h equilibration. There is no association between the sensitivity to cooling, based on the motility evaluation during storage at $15^{\circ} \mathrm{C}$, and the semen viability after freezing. A greater equilibration time (20h) before freezing allows the maintenance of higher acrosome integrity after thawing.

Key-words: acrosome, freezing, semen, spermatozoa, swine. 


\section{Introdução}

Aproximadamente $99 \%$ das 19 milhões de inseminações de suínos feitas anualmente no mundo (JOHNSON et al., 2000) empregam o sêmen resfriado a $15-18^{\circ} \mathrm{C}$ pelo fato da fertilidade ser similar à observada com monta natural (JOHNSON, 1998). Os baixos índices de fertilidade, os protocolos extremamente laboriosos e a variação na congelabilidade entre os doadores de sêmen têm dificultado o emprego rotineiro de sêmen congelado.

O congelamento do sêmen causa danos ao acrossomo, declínio da motilidade, perda da integridade da membrana plasmática e redução da atividade metabólica dos espermatozóides (ERIKSSON e RODRIGUEZ-MARTINEZ, 1996; HOLT e MEDRANO, 1997), levando à perda de sua capacidade fecundante. Com o emprego de sêmen congelado, os índices reprodutivos são insatisfatórios quando comparados aos obtidos com sêmen resfriado, observando-se reduções de 10 a $20 \%$ na taxa de parto e de 1 a 2 leitões por leitegada (HOFMO e ALMLID, 1991).

Pela grande sensibilidade dos espermatozóides suínos a temperaturas inferiores a $15^{\circ} \mathrm{C}$, um tempo mínimo de equilíbrio, acima dessa temperatura, é necessário para diminuir os efeitos deletérios do choque térmico e aumentar, assim, a viabilidade espermática após descongelamento (SALAMON, 1973). Efeito benéfico de um período prévio de incubação tem sido mostrado para sêmen resfriado a $5^{\circ} \mathrm{C}$ (WEITZE et al., 2000) ou congelado (KOTZIAS-BANDEIRA, 1997; ERIKSSON et al., 2001).

Para obter bons índices de produtividade, o uso do sêmen resfriado não deve ultrapassar três dias de armazenamento. Por isso, há interesse em desenvolver estudos para aperfeiçoar o protocolo de congelamento de sêmen suíno, de modo a obter maior viabilidade espermática após o descongelamento e, possivelmente, aumentar os índices de fertilidade in vivo.

O presente trabalho teve como objetivo estudar a influência da sensibilidade ao resfriamento e do tempo de equilíbrio précongelamento sobre a qualidade espermática após o congelamento.

\section{Material e Métodos}

O experimento foi conduzido no período de janeiro a julho de 2000, em uma central de inseminação climatizada, localizada no Estado de Santa Catarina. Nas instalações de coleta de sêmen e de alojamento dos machos, as médias de temperatura interna mínima e máxima foram de 21 e $25^{\circ} \mathrm{C}$, respectivamente.

Oito machos doadores de sêmen (híbridos de uma linhagem comercial composta por Landrace, Large White e Pietrain), com idade entre 20 e 42 meses, foram submetidos a coletas de sêmen com intervalos de três a quatro dias. Foram utilizados 10 ejaculados de cada macho. O sêmen foi mantido em banhomaria a $32^{\circ} \mathrm{C}$ e, por microscopia, fez-se a avaliação da motilidade, da concentração e da morfologia espermática. Os critérios utilizados para a escolha dos ejaculados foram os de motilidade superior a $75 \%$ e alterações morfológicas inferiores a $20 \%$.

Os ejaculados foram diluídos na proporção 1:1 com diluente BTS, a $32^{\circ} \mathrm{C}(50 \mathrm{~mL}$ de sêmen $+50 \mathrm{~mL}$ de Beltsville Thawing Solution (BTS). Cada ejaculado foi dividido em duas frações, uma delas foi submetida a um período de equilíbrio curto (1h e $30 \mathrm{~min}$; EC) e a outra a um período longo (20h; EL) a $20^{\circ} \mathrm{C}$, antes de serem resfriadas a $15^{\circ} \mathrm{C}$ por $2 \mathrm{~h}$ e $30 \mathrm{~min}$. As amostras foram congeladas com $2 \%$ de glicerol na concentração final e envase em palhetas de $0,5 \mathrm{~mL}$. O sêmen foi centrifugado $(800 \mathrm{~g}$ por 10 minutos, a $15^{\circ} \mathrm{C}$ ) e, ao final, o sobrenadante foi desprezado e o sedimento foi diluído com o diluente de resfriamento (DR - $80 \mathrm{~mL}$ de solução de lactose $11 \%$; $20 \mathrm{~mL}$ de gema de ovo). A concentração de espermatozóides no pellet foi estimada utilizando câmara hemocitométrica e, posteriormente, foi determinado o volume de ressuspensão do DR necessário para atingir a concentração de $1,5 \times 10^{9}$ espermatozóides $/ \mathrm{mL}$. Os espermatozóides foram mantidos em contato com o DR, a $5^{\circ} \mathrm{C}$, por $1 \mathrm{~h}$ e $30 \mathrm{~min}$, quando foi adicionado o diluente de congelamento (DC - $72,5 \mathrm{~mL}$ de lactose $11 \%$; $20 \mathrm{~mL}$ de gema de ovo; $6 \mathrm{~mL}$ de glicerol; $1,5 \mathrm{~mL}$ de Orvus-es-Paste), até atingir concentração final de $1,0 \times 10^{9}$ espermatozóides $/ \mathrm{mL}$. Imediatamente após ter sido acrescentado o DC, foi realizado o envase em palhetas médias 
Congelabilidade do sêmen suíno de acordo com o período de equilíbrio pré-congelamento e da sensibilidade...

$(0,5 \mathrm{~mL})$, as quais foram manualmente submetidas a vapor de nitrogênio $\left(-120^{\circ} \mathrm{C}\right)$, durante 20 minutos e, posteriormente, mergulhadas em nitrogênio líquido a $-196^{\circ} \mathrm{C}$.

As palhetas foram descongeladas em banhomaria a $37^{\circ} \mathrm{C}$ por 20 segundos e o seu conteúdo foi diluído (1:6) em BTS. Após 10 minutos a $37^{\circ} \mathrm{C}$, foram avaliados os percentuais de espermatozóides móveis (MOT), de acrossomos normais (NAR) e de membranas íntegras (MI). A MOT e NAR também foram avaliados após o teste de termoresistência (TTR), caracterizado pela incubação da amostra diluída, em banho-maria a $37^{\circ} \mathrm{C}$, por 2h (ALMLID e JOHNSON, 1988). Três amostras de cada palheta descongelada foram submetidas à avaliação da motilidade pelo método subjetivo de microscopia (100x). A morfologia do acrossomo foi avaliada a partir da mistura de $40 \mu \mathrm{L}$ de sêmen descongelado em $1 \mathrm{~mL}$ de formol citrato 2,9\%. Foram analisadas 200 células espermáticas ao microscópio óptico de contraste de fase (1000x), entre lâmina e lamínula.

A integridade das membranas espermáticas foi avaliada de acordo com o método de HARRISON e VICKERS (1990), com os corantes diacetato de carboxifluoresceína $(20 \mathrm{mM})$ e iodeto de propídio $(7,3 \mathrm{mM})$. As amostras foram incubadas por oito minutos a $30^{\circ} \mathrm{C}$ em banho-maria. Após esse período de incubação, foi examinada uma alíquota de $4 \mu \mathrm{l}$, entre lâmina e lamínula, em microscópio de epifluorescência (filtro $E X / D M / B F=450-490 /$ $510 / 520 \mathrm{~nm}$ ), em aumento de 1000x. Foram avaliadas 400 células por amostra, as quais foram classificadas conforme descrito por ORTMAN e RODRIGUEZ-MARTINEZ (1994).

A motilidade de doses de sêmen preparadas a partir de todos os ejaculados utilizados no congelamento, foi avaliada simultaneamente, durante o resfriamento a $15^{\circ} \mathrm{C}$, a cada $24 \mathrm{~h}$. Os ejaculados foram classificados, de acordo com a manutenção da motilidade, em dois grupos: motilidade $<60 \%$ (grupo E1) e motilidade $\geq 60 \%$ (grupo E2), no quinto dia de armazenamento a $15^{\circ} \mathrm{C}$.

A análise dos dados foi efetuada com o procedimento GLM (SAS, 1998). Foram considerados os efeitos do tempo de equilíbrio pré-congelamento, da sensibilidade dos ejaculados ao resfriamento, dos machos, e das interações duplas entre esses fatores. As médias foram calculadas pelo LSMeans e o teste $\mathrm{t}$, em nível de $5 \%$ de significância, foi utilizado para compará-las. Foi analisada a correlação entre a motilidade durante o resfriamento e a viabilidade após congelamento.

\section{Resultados e Discussão}

A motilidade média do sêmen após a diluição, antes da incubação e congelamento, foi $89,4 \pm$ 1,8. Após o descongelamento, os resultados médios de MOT, NAR e MI foram respectivamente 51,65 e $38 \%$, dentro das variações observadas por outros autores, no congelamento de sêmen suíno (ALMLID e JOHNSON, 1988; BERGER e FISCHERLEITNER, 1992; ORTMAN e RODRIGUEZ-MARTINEZ, 1994).

Não houve diferença entre os períodos de equilíbrio quanto à motilidade $(P>0,05)$, mas foi verificada superioridade $(P<0,05)$, quanto ao percentual de acrossomos normais, após o descongelamento e após teste TTR, nas amostras submetidas a $20 \mathrm{~h}$ de equilíbrio (TABELA 1). A ausência de efeito da interação entre o tempo de equilíbrio e a sensibilidade dos ejaculados $(P>0,05)$ mostra que o equilíbrio por 20h não trouxe maiores benefícios aos ejaculados menos resistentes ao resfriamento. Segundo PURSEL e PARK (1985), um tempo de equilíbrio é necessário antes de submeter os espermatozóides ao congelamento, de modo a diminuir os efeitos deletérios do choque térmico sobre os espermatozóides. KOTZIASBANDEIRA (1997), utilizando envase em macrotubos, verificou superioridade de $13 \%$ na motilidade e de $8 \%$ nos acrossomos normais, após o descongelamento, quando o tempo de equilíbrio a $20^{\circ} \mathrm{C}$ passou de $1 \mathrm{~h}$ e $30 \mathrm{~min}$ para $20 \mathrm{~h}$. Em relação à integridade das membranas, não foram detectadas diferenças entre os períodos de equilíbrio longo e curto, no presente estudo, contrariamente ao observado por ERIKSSON et al. (2001), os quais trabalharam com equilíbrio a $17^{\circ} \mathrm{C}$ e observaram aumentos significativos de $5 \%$ e $4 \%$ na integridade das membranas espermáticas, para os tempos de 10 e 20h, respectivamente, em relação ao grupo de $3 \mathrm{~h}$ a $17^{\circ} \mathrm{C}$. 
TABELA 1 - VIABILIDADE DE SÊMEN SUÍNO CONGELADO (PERCENTUAL MÉDIO \pm DESVIO-PADRÃO), DE ACORDO COM O TEMPO DE EQUILÍBRIO PRÉ-CONGELAMENTO E A SENSIBILIDADE DOS EJACULADOS AO RESFRIAMENTO.

\begin{tabular}{ccccc}
\hline $\begin{array}{c}\text { Características } \\
\text { de viabilidade } \\
\text { espermática }\end{array}$ & \multicolumn{2}{c}{$\begin{array}{c}\text { Tempo de } \\
\text { equilíbrio }\end{array}$} & \multicolumn{2}{c}{ Sensibilidade ao resfriamento } \\
\cline { 2 - 5 } & $\mathrm{EC}(\mathrm{n}=79)$ & $\mathrm{EL}(\mathrm{n}=80)$ & $\mathrm{E} 1(\mathrm{n}=93)$ & $\mathrm{E} 2(\mathrm{n}=66)$ \\
\hline $\begin{array}{c}\text { Motilidade pós- } \\
\text { descongelamento }\end{array}$ & $51,4 \pm 9,5$ & $51,4 \pm 9,9$ & $50,9 \pm 9,9$ & $51,9 \pm 9,2$ \\
$\begin{array}{c}\text { Motilidade pós- } \\
\text { TTR }\end{array}$ & $39,4 \pm 14,9$ & $41,4 \pm 11,6$ & $39,2 \pm 12,9$ & $41,6 \pm 13,6$ \\
$\begin{array}{c}\text { NAR pós- } \\
\text { descongelamento }\end{array}$ & $60,5 \pm 17,3 \mathrm{a}$ & $69,9 \pm 13,7 \mathrm{~b}$ & $65,5 \pm 16,4$ & $64,9 \pm 15,7$ \\
$\begin{array}{c}\text { NAR pós-TTR } \\
\text { MI pós- }\end{array}$ & $29,4 \pm 15,2 \mathrm{a}$ & $33,4 \pm 13,9 \mathrm{~b}$ & $30,9 \pm 14,1$ & $31,9 \pm 14,5$ \\
$\quad 39,3 \pm 12,7$ & $36,9 \pm 13,4$ & $37,0 \pm 13,1$ & $39,1 \pm 13,0$ \\
\hline descongelamento & & & & \\
\hline
\end{tabular}

Valores seguidos por letras distintas na linha, dentro do tempo de equilíbrio, diferem entre si $(P<0,05)$.

$\mathrm{NAR}=$ acrossomos normais; $\mathrm{Ml}=$ membranas íntegras.

$\mathrm{TTR}=$ teste de termoresistência.

$\mathrm{EC}=1 \mathrm{~h}$ e $30 \mathrm{~min}$ de equilíbrio a $20^{\circ} \mathrm{C}$.

$\mathrm{EL}=20 \mathrm{~h}$ de equilíbrio a $20^{\circ} \mathrm{C}$.

$\mathrm{E} 1=$ motilidade $<60 \%$ no quinto dia de armazenamento a $15^{\circ} \mathrm{C}$.

$\mathrm{E} 2=$ motilidade ${ }^{3} 60 \%$ no quinto dia de armazenamento a $15^{\circ} \mathrm{C}$.

O efeito do macho foi mantido no modelo de análise estatística pois o mesmo foi significativo para todos os itens de viabilidade espermática estudados. As diferenças entre machos quanto à viabilidade espermática após o congelamento confirmam observações anteriores (FISER et al., 1996; RODRIGUEZ-MARTINEZ et al., 1996; ROCA et al., 2000). A diferença na resistência do sêmen ao resfriamento entre machos suínos também é relatada (MARIANO, 1988; PAULENZ et al., 2000; REIS et al., 2002), embora sejam escassas as informações a respeito da relação entre a resistência ao resfriamento e a resistência ao congelamento. MARIANO (1988) relatou que nem todos os machos classificados como de boa resistência ao resfriamento ( $>50 \%$ de motilidade após $48 \mathrm{~h}$ a $15^{\circ} \mathrm{C}$ ) apresentaram boa resposta ao congelamento, sendo que alguns deles tiveram motilidade inferior a $30 \%$, após descongelamento. Considerando as possíveis variações que podem existir na resistência ao resfriamento entre ejaculados de um mesmo macho (REIS et al., 2002), no presente estudo os ejaculados foram agrupados de acordo com a capacidade de manter a motilidade durante 0 armazenamento, independentemente de que macho eram provenientes. Mesmo assim, os ejaculados de maior manutenção da motilidade durante o resfriamento a $15^{\circ} \mathrm{C}$, não diferiram na sua motilidade, integridade de membrana e de acrossomo, após descongelamento, daqueles mais sensíveis ao resfriamento. BERGER e FISCHERLEITNER (1992) não observaram correlação significativa da motilidade pósdescongelamento com a motilidade do sêmen in natura ou com o número de dias para que a motilidade do sêmen resfriado a $15^{\circ} \mathrm{C}$ atingisse um valor inferior a $30 \%$. No presente estudo, houve correlação significativa $(P<0,05)$ entre a motilidade nas $120 \mathrm{~h}$ e todos os parâmetros de viabilidade espermática, pós-congelamento, mas a mesma foi baixa, com coeficientes variando de 0,21 a 0,27.

$\mathrm{O}$ fato de machos apresentarem fertilidade 
Congelabilidade do sêmen suíno de acordo com o período de equilíbrio pré-congelamento e da sensibilidade...

nos índices comercialmente aceitáveis e responderem ao congelamento de forma diferente da prevista, dificulta o processo de seleção de doadores para o congelamento de sêmen. Em condições práticas, seria importante prever a resposta de um determinado macho frente ao congelamento. A comprovação da manutenção da resistência ao resfriamento nos ejaculados de um mesmo doador poderia auxiliar na escolha do macho cujo sêmen seria de boa congelabilidade. No entanto, a variação entre ejaculados de um mesmo macho e o fato dos ejaculados de boa resistência ao resfriamento não terem apresentado melhor comportamento após o congelamento, invalida essa possibilidade. A exposição dos espermatozóides a $10^{\circ} \mathrm{C}$, conforme sugerido por PAULENZ et al. (2000), ou mesmo temperaturas inferiores, talvez permitam evidenciar melhor a diferença na congelabilidade dos ejaculados ou dos machos, mas são necessários estudos nesse sentido.

\section{Conclusões}

O equilíbrio pré-congelamento por $20 \mathrm{~h}$ confere maior estabilidade ao acrossomo, após o descongelamento, em comparação ao equilíbrio por $1 \mathrm{~h}$ e $30 \mathrm{~min}$. O fato do período de equilíbrio de $20 \mathrm{~h}$ não ter acarretado danos espermáticos, abre a possibilidade de enviar as amostras de sêmen para que sejam congeladas em locais diferentes dos de coleta. A resistência do sêmen ao resfriamento, avaliada pela manutenção da motilidade a $15^{\circ} \mathrm{C}$, não permite prever a sua resposta ao congelamento.

\section{REFERÊNCIAS}

ALMLID; T.; JOHNSON, L.A. Effects of glycerol concentration, equilibration time and temperature of glycerol addition on post-thaw viability of boar spermatozoa frozen in straws. Journal of Animal Science, Savoy, v.66, p.2899-2905, 1988.

BERGER, B.; FISCHERLEITNER, F. On deepfreezing of boar semen: investigations on the effects of different straw volumes, methods of freezing and thawing extenders. Reproduction in Domestic Animals, Berlin, v.27, p.266-270, 1992.
ERIKSSON, B.; RODRIGUEZ-MARTINEZ, H. Assessment of membrane damage in frozen/thawed boar spermatozoa. Reproduction in Domestic Animals, Berlin, v.31, p.285-286, 1996.

ERIKSSON, B.; VAZQUEZ, J.M.; MARTINEZ, E.A.; ROCA, J.; LUCAS, X.; RODRIGUEZ-MARTINEZ, $\mathrm{H}$. Effects of holding time during cooling and of type of package on plasma membrane integrity, motility and in vitro oocyte penetration ability of frozenthawed boar spermatozoa. Theriogenology, New York, v.55, p.1593-1605, 2001.

FISER, P.S.; FAIRFULL, R.W.; PANICH, P.L. Glycerol equilibration time revisited. Reproduction in Domestic Animals, Berlin, v.31, p.141-146, 1996.

HARRISON, R.A.P.; VICKERS, S.E. Use of fluorescent probes to assess membrane integrity in mammalian spermatozoa. Journal of Reproduction and Fertility, Cambridge, v.88, p.343-352, 1990.

HOFMO, P.O.; ALMLID, T. Recent developments in freezing of boar semen with special emphasis on cryoprotectans. Reproduction in Domestic Animals, Berlin, Suppl.1, p.111-122. 1991.

HOLT, W.V.; MEDRANO, A. Assessment of boar sperm function in relation to freezing and storage. Journal of Reproduction and Fertility, Cambridge, Suppl.52, p.213-222, 1997.

JOHNSON, L.A. Current developments in swine semen: preservation, artificial insemination and sperm sexing. In: INTERNATIONAL PROCEEDINGS OF VETERINARY SWINE CONGRESS, 5, 1998, Birmingham-England. Proceedings... Wiltshire, 1998. v.1, p.225-229.

JOHNSON, L.A.; WEITZE, K.F.; FISER, P.; MAXWELL, W.M. Storage of boar semen. Animal Reproduction Science, Amsterdam, v.62, p.143-172, 2000.

KOTZIAS-BANDEIRA, E. Auswirkung von Kurzund Langzeitäquilibrierung vor der Gefrierung von Ebersperma auf Auftauqualität und Membranzustand der Samenzellen. Hannover, 1997. 97p. Tese (Doutorado em Reprodução de Suínos) - Escola Superior de Veterinária de Hannover.

MARIANO, M.S. Características biológicas e bioquímicas do sêmen suíno com diferentes resistências à conservação no estado líquido e ao congelamento. Santa Maria, 1988. 72f. Dissertação (Mestrado em Ciências Veterinárias) - Curso de Medicina Veterinária, Universidade Federal de Santa Maria.

ORTMAN, K.; RODRIGUEZ-MARTINEZ, H. Membrane damage during dilution, cooling and freezing-thawing of boar spermatozoa packaged in plastic bags. Journal of Veterinary Medicine, Berlin, v.41, p.37-47, 1994. 
PAULENZ, H.; KOMMISRUD, E.; HOFMO, P.O. Effect of long-term storage at different temperatures on the quality of liquid boar semen. Reproduction in Domestic Animals, Berlin, v.35, p.83-87, 2000.

PURSEL, V.G.; PARK, S. Freezing and thawing procedures for boar spermatozoa. In: INTERNATIONAL CONFERENCE ON DEEP FREEZING OF BOAR SEMEN, 1, 1985, Uppsala. Proceedings... Uppsala, 1985. p.147-166.

REIS, G.R.; BERNARDI, M.L.; SCHWARZ, P.; BORTOLOZZO, F.P.; WENTZ, I. Diferença entre machos suínos na manutenção da viabilidade espermática a $17^{\circ} \mathrm{C}$. Acta Scientiae Veterinariae, Porto Alegre, v.30, n.3, p.159-166, 2002.

ROCA, J.; LUCAS, M.A.; GIL, J.M.; VAZQUEZ, G. Motility and in vitro penetrating ability of cooled and frozen thawed spermatozoa from identical boars. In: INTERNATIONAL CONFERENCE ON BOAR SEMEN PRESERVATION, 4, 1999, Beltsville, Maryland, USA. Anais... Lawrence, Allen, 2000. p.260.

Recebido para publicação: 03/07/2004 Aprovado:
RODRIGUEZ-MARTINEZ, H.; ERIKSSON, B.; LUNDEHEIN, N. Freezing boar semen in flat plastic bags membrane integrity and fertility. Reproduction in Domestic Animals, Berlin, v.31, p.161-168, 1996. SALAMON, S. Deep freezing of boar semen. III Effects of centrifugation, diluent and dilution rate, pellet volume, and method of thawing on survival of spermatozoa. Australian Journal of Biological Science, Collingwood, v.26, p.239247, 1973.

SAS INSTITUTE. SAS user's guide: Statistical Analysis System, Release 6.12, Cary NC: SAS Institute Inc, 1998.

WEITZE, K.F.; WEBER, H.; WABERSKI, D. Influence of incubation time and cooling rate on chilling sensitivity of diluted boar semen. In: INTERNATIONAL CONFERENCE ON BOAR SEMEN PRESERVATION, 4, 1999, Beltsville, Maryland, USA. Anais... Lawrence, Allen, 2000. p.264. 